\title{
Multisystem Inflammatory Syndrome with Features of Incomplete Kawasaki Disease in an Adolescent Boy with COVID-19
}

\author{
Pallithottangal Kunju Moideen Hidayathullah,, (D) Shamsudeen Moideen, ${ }^{2}$ \\ (1) Santha Velappan Rakhesh, ${ }^{3}$ (1) Pichen Shihabudheen, ${ }^{4}$ (1) Nalakath Arakkal Uvais ${ }^{5}$ \\ 'Department of Paediatrics, Iqraa International Hospital and Research Centre, Calicut, Kerala, India \\ ${ }^{2}$ Department of Internal Medicine, Iqraa International Hospital and Research Centre, Calicut, \\ Kerala, India \\ ${ }^{3}$ Department of Dermatology, Iqraa International Hospital and Research Centre, Calicut, Kerala, India \\ ${ }^{4}$ Department of Critical Care, Iqraa International Hospital and Research Centre, Calicut, Kerala, India \\ ${ }^{5}$ Department of Health Research, Iqraa International Hospital and Research Centre, Calicut, \\ Kerala, India
}

\section{ABSTRACT}

Although the coronavirus disease (COVID-19) in the pediatric population appears to be less severe among children $<18$ years old, emerging evidence from Europe, North America, Asia, and Latin America indicates that COVID-19 infection may precipitate the hyperinflammatory state among children following infection. This paper reports a case of multisystem inflammatory syndrome with features of incomplete Kawasaki disease in an adolescent boy who also tested positive for COVID-19 antibody. This case highlights the potential COVID-19 complications in adolescents.

Keywords: Adolescents, COVID-19, Kawasaki disease, MIS-C associated with COVID-19

\section{INTRODUCTION}

While the coronavirus disease (COVID-19) appears to be less severe among children $<18$ years old, emerging evidence from Europe, North America, Asia, and Latin America indicates that COVID-19 infection may precipitate the hyperinflammatory state among children following infection. ${ }^{[1]}$ Multiple reports have highlighted the association between multisystem inflammatory syndrome in children and COVID-19 infection. ${ }^{[2,3]}$ Not only children but also adolescents are presented with the same syndrome. Many of them with additional features of Kawasaki disease (KD). ${ }^{[4]}$ The following case describes the clinical features, treatment, and response of an adolescent boy from South India who presented with a KD-like inflammatory disorder with evidence of a prior COVID-19 infection.

\section{CASE REPORT}

A 17-year-old boy was admitted with multiple swelling in the neck for 10 days. The swellings were accidentally noticed, which started at the right side of the neck followed by the left side on the next day. The swellings were gradually increasing and were painful. An associated sore 
throat and dysphagia were initially noted. The patient started having acute high-grade fever 1 week previously with chills and riggers, which was acute in onset, aggravated during the night with sweating. The fever was relieved after taking medications. In addition, the patient also developed diarrhea, which was not foul-smelling, not blood-stained, and was associated with abdominal pain, 3 days before hospital admission. One day before the patient's admission to the hospital, the patient noticed erythematous rashes at his back, which gradually extended to the chest, upper limbs, and lower limbs. No associated itching was noted. However, the patient also reported scrotal pain which was associated with swelling and erythema. No history of vomiting, palpitation, headache, breathing difficulty, giddiness, and conjunctivitis was reported. Furthermore, no history of type 2 diabetes mellitus, hypertension, heart disease, tuberculosis, rheumatic fever, jaundice, surgery, and previous allergy was noted. The patient also had no history of recent travel and any exposure to any sexually transmitted diseases. Moreover, no similar illness was noted among members of the patient's family. A history of exposure to a COVID19-infected patient 2 weeks ago was highlighted, and the patient was tested for COVID-19 antigen. However, the results were negative. The patient was found to be moderately built and nourished with cervical lymphadenopathy (levels 2 and 3 on the right side; levels 2, 3, and 4 on the left side) and with maculopapular rashes in the chest, back, and upper and lower limbs on examination. The heart rate, blood pressure, and respiratory rate of the patient were $110 / \mathrm{min}, 90 / 50 \mathrm{mmHg}$, and $22 / \mathrm{min}$, respectively. Systemic examinations were normal. The patient clinically met the criteria for incomplete KD.

Leukocytosis, elevated inflammatory markers such as, Creactive protein (CRP) and procalcitonin, hyponatremia, hypoalbuminemia, elevated liver enzymes, troponins and, D-dimer, and negative for immunologic (IMN) serology were detected in the initial laboratory measurements. Initial laboratory measurements of the patient are summarized in Table 1. Chest X-ray showed an enlarged cardiac silhouette. An echocardiogram showed global left ventricular hypokinesia with mild left ventricular systolic dysfunction. However, no ectasia, dilation, or aneurysm formations of coronary arteries were found. However, the patient's antibody test for COVID-19 was positive and he was transferred to the intensive care unit. Consequently, the patient was treated with intravenous immunoglobulin (IVIG) therapy (2 $\mathrm{g} / \mathrm{kg})$, methylprednisolone $(5 \mathrm{mg} / \mathrm{kg}$ ), and aspirin (2 $\mathrm{mg} / \mathrm{kg}$ ). The patient experienced significant resolution of symptoms with no fever, no dyspnea, normal vital signs, lowering of CRP and D-dimer levels, and rash disappearance within 1 week of admission.

\begin{tabular}{lcc}
\multicolumn{3}{l}{ Table 1. Initial laboratory measurements of the patient } \\
& Value & $\begin{array}{c}\text { Reference } \\
\text { values }\end{array}$ \\
& & $4500-11000$ \\
\hline White blood cells (cells/cumm) & 16900 & $4.0-10.0$ \\
C-reactive protein (mg/L) & 93.6 & $\leq 0.15$ \\
Procalcitonin (ng/mL) & 44.32 & $135.0-145.0$ \\
Serum sodium (mmol/L) & 131.0 & $3.4-5.4$ \\
Serum albumin $(\mathrm{g} / \mathrm{dL})$ & 2.8 & $29.0-33.0$ \\
Alanine aminotransferase (U/L) & 50.0 & $13.5-14.5$ \\
Troponins (pg/mL) & 8901.1 & $<250.0$ \\
D-dimer (ng/mL) & 5618.94 & $140.0-280.0$ \\
Lactate dehydrogenase (U/L) & 247.0 & $<125.0$ \\
ProB-type natriuretic peptide $(\mathrm{pg} / \mathrm{mL})$ & $>25000$ & $20.0-250.0$ \\
Ferritin (ng/mL) & 1702.56 & \\
\hline
\end{tabular}

\section{DISCUSSION}

The patient was diagnosed with a multisystem inflammatory disorder of children and adolescents with features similar to incomplete KD based on the following features: fever $>3$ days, rash, myocardial dysfunction (elevated troponin/ NT), coagulopathy (elevated D-dimers), acute gastrointestinal problems, and elevated markers of inflammation. In the current case, reverse transcription-polymerase chain reaction (RT-PCR) was negative on two occasions. However, the patient had a history of exposure to a COVID-19 positive case and he tested positive in the antibody tests indicating a previous asymptomatic COVID-19 infection, which is a common finding among children and adolescents. ${ }^{[5]}$ Furthermore, Rauf et al. reported a similar case from the same city where the hospital of the current study is located in a 5-year-old child wherein real-time PCR for severe acute respiratory syndrome coronavirus 2 (SARS-CoV-2) was done and resulted negative twice. ${ }^{[6]}$ However, an antibody test could not be done at that time due to its unavailability. Similar reports were also reported from the UK. ${ }^{[7]}$

$\mathrm{KD}$ is seasonal and rare but is a potentially severe inflammatory condition mostly occurring in $>5$-year-old children. ${ }^{[8]}$ Some evidence suggests that multiple factors play different roles in KD pathogenesis (e.g., genetic predisposition, autoimmunity, infectious etiology, and host immune and inflammatory response) ${ }^{[9]}$ Different explanations could exist for the possible association between COVID infection and KD. Most of the cases reported have undetectable SARS-CoV-2 on RT-PCR and only a few cases have lgG antibodies for the COVID-19 virus. It suggests that a coronavirus infection in the past or contact with a COVID-19 person can trigger an immune response simulating $K D{ }^{[6,10]} \mathrm{A}$ recent review outlined the possible mechanisms for an acquired immune response to accentuate SARS-CoV-2 which 
includes an antibody or T-cell recognition of self-antigens resulting in autoantibodies, anti-body, or T-cell recognition of viral antigens expressed on infected cells, immune complexes formation, and viral superantigen sequences which activate the host immune cells. ${ }^{[1]}$

The multisystem inflammatory disorder in children (MISC) and adolescents with features similar to incomplete $\mathrm{KD}$ is an evolving disease and the true pathophysiology is yet unknown. However, it can be safely argued that MIS-C shares some common etiology and pathophysiology with $\mathrm{KD}$ based on existing evidence regarding clinical presentation and laboratory investigation. Many differences were also noted between these two conditions (e.g., age of presentation, pattern of cardiac involvement, racial differences, and so on). ${ }^{[10]}$

Reports indicate that MIS-C is less common in Asia compared with US and European countries. However, a recent study reported from Pakistan described eight children with MIS-C. All the children were asymptomatic, male, with a median age of 9.5 years, and with positive COVID-19 antibody tests. ${ }^{[3]}$ Three patients also had a positive PCR for SARS-CoV-2, and two-thirds of children also showed coronary artery dilatations. However, similar to the patient of the current study, hypotension and shock were less common among them.

\section{CONCLUSION}

The current case represents an early report of a KD-like illness in an adolescent from Asia with serologic evidence of a previous COVID-19 infection. Understanding of this condition is limited, but rapidly evolving. Thus, the current report demands that physicians should be more aware of the secondary inflammatory syndromes that mimic KD among adolescents following asymptomatic COVID-19 infection so that it can be diagnosed early and treated promptly with IVIG and steroids for good prognosis.

\section{Disclosures}

Informed Consent: Written informed consent was obtained from the patient's family to publish the case report.

Conflict of Interest: The authors did not report any conflict of interest on this manuscript.

Peer-review: Externally peer-reviewed.
Authorship contributions: Concept - P.K.M.H., S.M., S.V.K., S.P., N.A.U.; Design - P.K.M.H., S.M., S.V.K., S.P., N.A.U.; Supervision P.K.M.H., S.M., S.V.K., S.P., N.A.U.; Materials - P.K.M.H., S.M., S.V.K., S.P., N.A.U.; Data collection \&/or processing - P.K.M.H., S.M., S.V.K., S.P., N.A.U.; Analysis and/ or interpretation - P.K.M.H., S.M., S.V.K., S.P., N.A.U.; Literature search - P.K.M.H., S.M., S.V.K., S.P., N.A.U.; Writing - P.K.M.H., S.M., S.V.K., S.P., N.A.U.; Critical Review - P.K.M.H., S.M., S.V.K., S.P., N.A.U.

\section{REFERENCES}

1. Jiang L, Tang K, Levin M, Irfan O, Morris SK, Wilson K, et al. COVID-19 and multisystem inflammatory syndrome in children and adolescents. Lancet Infect Dis 2020;20(11):276-88. [CrossRef]

2. Feldstein LR, Rose EB, Horwitz SM, Collins JP, Newhams MM, Son MBF, et al. Multisystem inflammatory syndrome in U.S. children and adolescents. N Engl J Med 2020;383(4):334-46.

3. Sadiq M, Aziz OA, Kazmi U, Hyder N, Sarwar M, Sultana N, et al. Multisystem inflammatory syndrome associated with $\mathrm{CO}$ VID-19 in children in Pakistan. Lancet Child Adolesc Health 2020;4(10):36-7. [CrossRef]

4. Ouldali N, Pouletty M, Mariani P, Beyler C, Blachier A, Bonacorsi $\mathrm{S}$, et al. Emergence of Kawasaki disease related to SARS-CoV-2 infection in an epicentre of the French COVID-19 epidemic: a time-series analysis. Lancet Child Adolesc Health 2020;4:66268. [CrossRef]

5. Lu X, Zhang L, Du H, Zhang J, LiYY, Qu J, et al. SARS-CoV-2 infection in children. N Engl J Med 2020;382(17):1663-5. [CrossRef]

6. Rauf A, Vijayan A, John ST, Krishnan R, Latheef A. Multisystem inflammatory syndrome with features of atypical Kawasaki disease during COVID-19 pandemic. Indian J Pediatr 2020;87(9):745-7. [CrossRef]

7. Riphagen S, Gomez X, Gonzalez-Martinez C, Wilkinson N, Theocharis P. Hyperinflammatory shock in children during COVID-19 pandemic. Lancet 2020;395(10237):1607-8. [CrossRef]

8. Shah SK, Munoz AC. Multisystem inflammatory syndrome in children in COVID-19 pandemic. Indian J Pediatr 2020;87:6713. [CrossRef]

9. Raba AA, Abobaker A. COVID-19 and Kawasaki disease: An etiology or coincidental infection? Pediatr Infect Dis J 2020;39(8):e213. [CrossRef]

10. Verdoni L, Mazza A, Gervasoni A, Martelli L, Ruggeri M, Ciuffreda $M$, et al. An outbreak of severe Kawasaki-like disease at the Italian epicentre of the SARS-CoV-2 epidemic: an observational cohort study. Lancet 2020;395(10239):1771-8. [CrossRef] 